Ordonhes, M.T.; López-Gil, J.F.; Caregnato, A.F.; Cavichiolli, F.R. (2021) Analysis of the Continuity of Brazilian Swimmers in the Bolsa-Atleta Program. Revista Internacional de Medicina y Ciencias de la Actividad Física y el Deporte vol. 21 (84) pp. 419-434. Http://cdeporte.rediris.es/revista/revista83/artanalisis1265.htm

DOI: https://doi.org/10.15366/rimcafd2021.83.001

\title{
ORIGINAL
}

\section{ANÁLISIS DE LA CONTINUIDAD DE LOS NADADORES BRASILEÑOS EN EL PROGRAMA BOLSA-ATLETA}

\author{
ANALYSIS OF THE CONTINUITY OF BRAZILIAN \\ SWIMMERS IN THE BOLSA-ATLETA PROGRAM

\section{ANÁLISE DA CONTINUIDADE DOS NADADORES BRASILEIROS NO PROGRAMA BOLSA ATLETA}

\author{
Ordonhes, M.T.1; López-Gil, J.F.' ${ }^{2}$ Caregnato, A.F. ${ }^{2}$ y Cavichiolli, F.R. ${ }^{2}$ \\ ${ }^{1}$ Doctoranda en Educación Física. Universidad Federal de Paraná. Curitiba, Paraná (Brasil) \\ mayaraordonhes@hotmail.com \\ 2 Doctor en Educación Física. Universidad Federal de Paraná. Curitiba, Paraná (Brasil) \\ josefranciscolopezgil@gmail.com, andre.caregnato@hotmail.com, cavicca@hotmail.com
}

Traductor portugués-español: José Francisco López-Gil, josefranciscolopezgil@gmail.com

\section{AGRADECIMIENTOS}

El presente trabajo fue desarrollado con el apoyo de la Coordinación de Perfeccionamiento de Personal de Nivel Superior (CAPES) (Brasil), además del Proyecto Inteligencia Deportiva, fruto de la asociación entre el Ministerio de la Ciudadanía y la Universidad Federal de Paraná (UFPR) (Brasil).

Código UNESCO I UNESCO code: 5999 Otras Especialidades Políticas (Política Deportiva) / Other Political Specialties (Sports Policy).

Clasificación Consejo de Europa / Council of Europe classification: 1. Administración organización y gestión del deporte / Administration and management of sport organization.

Recibido 1 de julio de 2019 Received July 1, 2019

Aceptado 24 de abril de 2020 Accepted April 24, 2020

\section{RESUMEN}

El presente estudio tuvo por objetivo identificar, a partir del Programa BolsaAtleta desarrollado por el gobierno brasileño, si los nadadores que reciben incentivo financiero en las categorías de base, presentaron continuidad en la práctica deportiva hasta las categorías más altas del programa. Se estableció un 
recorte temporal de 2005 y 2017; siendo el número total de la muestra de 2.308 becas y de 1.017 atletas. Asimismo, todos los atletas seleccionados pertenecían a la modalidad de natación. La continuidad de los atletas en la recepción de becas presentó diferencias significativas en las respectivas categorías del programa $\left(p<0,001 ; \varepsilon^{2}=0,15\right)$. Se observaron diferencias significativas entre las becas de las categorías base e internacional, con un tamaño de efecto grande $(p<0,001 ; r=0,91)$. Finalmente, se puede afirmar que la inversión dirigida a las categorías de base no representa, de manera inexorable, la continuidad de los atletas en el desarrollo deportivo.

PALABRAS CLAVE: Políticas Públicas; Financiamiento Público; Gestión de Recursos; Deportes Acuáticos.

\section{ABSTRACT}

The objective of this study was to identify, from the Bolsa-Atleta Program developed by the Brazilian government, if the swimmers who received financial incentives in the base categories presented continuity in sports practice up to the highest categories of the program. The studied period was between 2005 and 2017; including 2,308 grants and 1,017 athletes. All the athletes selected belonged to the swimming modality. The continuity of the athletes in receiving scholarships showed significant differences in the respective categories of the program $\left(p<0,001 ; \varepsilon^{2}=0,15\right)$. Significant differences were found for the grants between the base and international categories, with a strong effect size $(p<0,001 ; r=0,91)$. Finally, it can be concluded that the investment directed to the base categories does not represent the continuity of the athletes in sports development.

KEYWORDS: Public Policies; Financing, Government; Resources Management; Water Sports.

\section{INTRODUCCIÓN}

La presencia del fenómeno deportivo en la agenda política de los países es debida a diversos motivos, como son: aspectos relacionados con la salud y la promoción de estilos de vida saludables (Hernández-Álvarez, VelázquezBuendía, Martínez-Gorroño y Díaz del Cueto, 2010); la promoción, organización y gestión de actividades deportivas (Gimeno, Garzo, Velasco, Sáenz y Gutiérrez, 2017; Fernández, Couto y Aza, 2016); o la capacidad de promoción de identidad nacional en una nación (Green y Oakley, 2001, Houlihan y Green, 2008).

Numerosos estudios han sido elaborados a partir de comparaciones entre las distintas naciones en aras de identificar los respectivos sistemas deportivos de los países (De Bosscher, De Knop, Van Bottenburg y Shibli, 2006; De Bosscher, Bingham, Shibli, Van Bottengurg y De Knop, 2008; De Bosscher, De Knop, Van Bottenburg, Shibli y Bingham, 2009; Digel, 2002; Green y Oakley, 2001). Tales comparaciones fueron realizadas considerando la posibilidad de que las naciones pudieran aprender unas de otras (Houlihan y Green, 2008); hecho que 
se ha traducido en la existencia de sistemas deportivos cada vez más homogéneos (Green y Oakley, 2001).

Asimismo, se puede observar en dichos estudios que los gobiernos y las organizaciones deportivas han aumentado las inversiones dirigidos al deporte de rendimiento (Seguí-Urbaneja, Inglés, Alcaraz y De Bosscher, 2020; Leiva, Sánchez y Martínez, en prensa; De Bosscher, De Knop, Van Bottenburg \& Shibli, 2006; De Bosscher, Bingham, et al. 2008; De Bosscher, De Knop, Van Bottenburg, Shibli \& Bingham, 2009; Digel, 2002; Green y Oakley, 2001). De igual modo, Leiva Arcas, Sánchez Pato y Martínez (en prensa) afirman que la racionalización de las inversiones puede aumentar las posibilidades de obtener resultados deportivos significativos.

Además, puede percibirse que la inversión financiera representa un importante factor contextual relacionado con la formación de atletas. En los gobiernos cuyo desarrollo deportivo ocurre a partir de la asociación entre la financiación pública y privada, este direccionamiento de recursos para el deporte sucede a partir de la formulación, implementación y evaluación de políticas públicas deportivas.

En otro orden de ideas, el factor financiero no es el único determinante para la formación de atletas; interviniendo otros factores influyentes en este proceso. Así, aparecen el efecto relativo de la edad, el género, los factores antropométricos y fisiológicos, las habilidades, las características psicológicas y las orientaciones motivacionales, los rasgos de personalidad, el lugar de nacimiento, los aspectos sociales, el volumen de entrenamientos específicos del deporte, la influencia de la especialización precoz, además de las características específicas de la modalidad de natación como, por ejemplo, el costo elevado que es necesario para la práctica en el país y la necesidad de infraestructura deportiva; postulándose todos ellos como factores de relevancia (Martínez de Ojeda, Puente-Maxera y Méndez-Giménez, en prensa; Caetano, Sentone, López-Gil, Caetano y Cavichiolli; 2020; Seguí-Urbaneja, et al., 2020; Sentone, López-Gil, Caetano y Cavichiolli, 2019; Barker-Ruchti et al., 2018; Blázquez y Feu, 2012; Côté y Hancock, 2016; Rees et al., 2016).

Sin embargo, la estructura financiera posee un carácter determinante en un sistema de rendimiento deportivo (Digel, 2002); pudiendo ser considerada como un factor vital para todo el proceso cuando el objetivo es perseguir unos buenos resultados, apoyando a la eficiencia y eficacia de los demás actores en el proceso de formación deportiva (De Bosscher, De Knop, Van Bottenburg \& Shibli, 2006; De Bosscher, Bingham, et al. 2008; De Bosscher, De Knop, Van Bottenburg, Shibli \& Bingham, 2009).

En el estudio realizado por Hollings, Mallett y Hume (2014), se ha evidenciado que algunos atletas poseen demandas sociales concurrentes con el deporte (familia, empleo, foco en la vida académica, búsqueda por otra carrera, etc.); siendo estas demandas, en conjunción con la falta de soporte en la carrera deportiva, factores que influyen para que éstos no evolucionen hasta niveles internacionales. 
En muchas organizaciones gubernamentales, la gestión del desarrollo de atletas ocurre a través de la distribución estratégica de fondos para deportes que puedan recuperar la inversión a través de resultados deportivos (Barker-Ruchti et al., 2018). Aunque los atletas de base son capaces de presentar resultados deportivos significantes, el proceso de formulación de una política pública deportiva debe preservar los beneficios de la práctica deportiva, resguardando las necesidades de los atletas, y contribuyendo al aumento de la participación y mantenimiento del desempeño futuro; es decir, evitando que el direccionamiento de recursos sea pautado por metas a corto plazo (Côté y Hancock, 2016, Bosscher, Shibli y Weber, 2019). Para Barker-Ruchti et al. (2018), aunque la organización de recursos públicos para atletas sea cada vez más amplia, ésta no garantiza el desarrollo de atletas a largo plazo (Hollings, Mallett y Hume, 2014, Huxlei, O'Connor y Bennie, 2018). Además, la relación entre el deporte de élite y el deporte de base se ha discutido continuamente en la literatura (SeguíUrbaneja et al., 2020).

Tales antecedentes teóricos presentaron diversos aspectos influyentes en la organización de recursos (Barker-Ruchti et al., 2018, Côté y Hancock, 2016, Feiler et al., 2018, Murray, 2009, Rees et al., 2016, Wicker et al., 2012). Sin embargo, no fue posible localizar estudios que tratasen de identificar si la inversión financiera dirigida a las categorías de base, representa o no, la garantía de la continuidad de los atletas en el desarrollo deportivo del país. De este modo, podemos observar una carencia de trabajos de investigación relacionados con esta temática; pudiendo ser percibida la importancia del desarrollo de investigaciones que analicen la continuidad de los atletas en los programas de incentivo financiero.

\section{OBJETIVOS}

La finalidad de este estudio fue identificar, a partir del ejemplo de una política pública deportiva (en esto caso, la propia de Brasil), si los nadadores que reciben incentivo financiero en las categorías iniciales, denominadas categorías de base, presentan continuidad en el rendimiento deportivo hasta las categorías de financiamiento más altas del programa (internacional y olímpica).

La realización del estudio se justifica teniendo en cuenta que, de este modo, se puede analizar y cuestionar la forma en que las políticas públicas se desarrollan y si las mismas se asocian a un desarrollo de los atletas a largo plazo.

\section{MATERIAL Y MÉTODOS}

\section{DISEÑO DEL ESTUDIO Y MUESTRA}

La investigación consiste en un estudio descriptivo ex post facto, teniendo por objeto relatar determinados acontecimientos, utilizando métodos comparativos y correlacionales para la ejecución del análisis (Kothari, 2004).

Se utilizó como muestra los atletas brasileños contemplados por el Programa Bolsa-Atleta entre los años 2005 y 2017 en la modalidad deportiva de natación. 
Asimismo, el presente estudio contó con la aprobación del Comité de Ética de la Universidade Federal do Paraná (UFPR) (CAAE nº 88770618.4.0000.0102).

Se repartieron un total de 2.308 becas, entre 1.017 atletas, siendo $57,62 \%$ del sexo masculino $(n=586)$ y $42,38 \%$ del sexo femenino $(n=431)$. Se analizaron las siguientes categorías del programa Bolsa-Atleta: categorías de base (base y estudiantil, $n=669)$, nacional $(n=1.285)$, internacional $(n=277)$ y olímpica $(n=77)$.

\section{PROCEDIMIENTOS}

El Programa Bolsa-Atleta fue creado en 2004 y tiene como objetivo ayudar mediante becas económicas a aquellos atletas brasileños que muestran resultados significativos en el año anterior a la presentación de la solicitud de la misma (Brasil, 2020). Las informaciones documentales referentes al programa fueron obtenidas por medio de las listas de atletas pertenecientes al programa, divulgadas por el Gobierno Federal de Brasil y publicadas en el Diario Oficial de la Unión (DOU) (Diário Oficial da União, 2020). Se estableció el recorte temporal de 2005 a 2017. El año 2005, por ser el primer año de concesión, y el año 2017 por ser el último año con los datos disponibles para la realización de este trabajo de investigación (Inteligência Esportiva, 2018).

Los datos fueron tabulados en función de las siguientes categorías y grupos: año de contemplación $(n=13,2005$ hasta 2017), atleta $(n=1.017)$, género $(n=2$, mujeres y hombres), categoría de la beca ( $n=4$; base, nacional, internacional, olímpica), valor económico anual ( $n=4, \$ 1.116$ USD, \$ 2.784 USD, \$ 5.556 USD, \$ 9.312 USD), valor económico mensual ( $n=4, \$ 93$ USD, \$ 232 USD, \$ 463 USD, $\$ 776$ USD), continuidad ( $n=9,1-9$ años). Se consideró como continuidad la frecuencia de la recepción de becas en el recorte temporal analizado.

Para la realización del análisis de la distribución de las becas, fueron seleccionados los nadadores que recibieron becas en las categorías de formación (base y estudiantil). Posteriormente, fueron identificados cuáles de estos atletas progresaron en las categorías de becas en el programa. Además del análisis de la progresión de los atletas, también se realizó el análisis inverso, es decir, se identificaron qué atletas recibieron becas en las categorías Olímpicas e Internacionales, y de éstos, cuantos retrocedieron hacia categorías inferiores del programa. El proceso inverso (retroceso) se justifica por identificar qué atletas recibieron sólo categorías más altas del programa Bolsa-Atleta, sin antes haber recibido categorías menores.

\section{ANÁLISIS DE LOS DATOS}

Después de la tabulación de los datos a través del software SPSS (Statistical for the Social Sciences), se realizaron análisis estadísticos relacionados con la continuidad de los atletas incluidos entre los años 2005 y 2017.

En primera instancia, se realizó la prueba de Kolmogorov-Smirnov, para verificar el cumplimento del supuesto de normalidad de los datos. Con el fin de evaluar la existencia de asociación entre las variables y comparar las diferencias existentes 
en las muestras, se realizaron las siguientes pruebas estadísticas no paramétricas: $H$ de Kruskal-Wallis, $U$ de Mann-Whitney, además de análisis post hoc con comparaciones pairwise y corrección de Bonferroni para la significancia. Por último, para potenciar la discusión y evitar posibles interferencias del tamaño de la muestra en los resultados, se calculó la magnitud del efecto a partir del coeficiente épsilon al cuadrado $\left(\varepsilon^{2}\right)$ (Kelley, 1935; Tomczak, Tomczak, 2014), así como $r$ de Rosenthal (Rosenthal, 1991); con la interpretación sugerida por Cohen (1988).

Todos los análisis se realizaron a través del software SPSS en su versión 24 para Windows y Microsoft Excel 2016; manteniendo el nivel de significancia estadística en $p<0,050$.

\section{RESULTADOS}

A partir del análisis de la distribución de los datos, se verificó el no cumplimiento del supuesto de normalidad para la continuidad, categoría de beca y valores anuales y mensuales en la modalidad de natación entre los años 2005 y 2017 $(p<0,05)$.

Desde 2005, su primer año de vigencia, el Programa Bolsa-Atleta destinó becas para atletas de diversas modalidades, tanto colectivas como individuales. El número total de becas concedidas para atletas de natación entre 2005 y 2017 fue de 2.308, subdivididas entre las diferentes categorías, donde: 669 becas fueron de formación (323 de la categoría base y 346 de la categoría estudiantil), 1.285 becas nacionales, 277 becas internacionales y 77 becas olímpicas. En la Figura 1, es posible observar el número de becas en cada año de concesión.

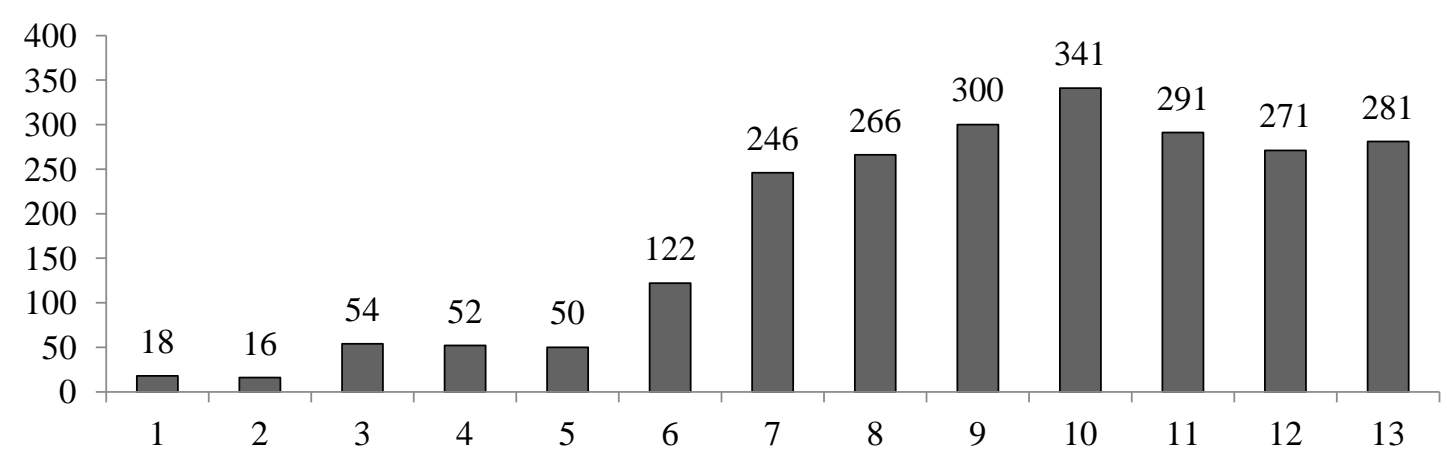

Figura 1. Número de concesiones para la natación entre 2005 y 2017.

En relación a la modalidad de natación, se perciben dos momentos diferenciados en razón a la cantidad de becas concedidas. El primero, entre los años 2005 a 2009; siendo el año 2007 el de mayor número de concesiones (54). El segundo período diferenciado comprende el período desde el año 2010 hasta el 2017, donde el número asignado de becas aumentó de manera significativa.

Por otra parte, la mayor diferencia está entre 2009 y 2010. En 2009, fueron entregadas un total de 50 becas. Este número de becas aumentó hasta 122 becas en 2010, representando un aumento del 144\% ( $n=72)$. De 2010 a 2011, 
este aumento fue del 102\% ( $n=124)$, alcanzando el número de becas su valor más elevado en 2014, donde se repartieron 341 becas por el programa. A continuación, la Figura 2 muestra el número de becas entregadas por categoría y por año, en la modalidad deportiva de natación.

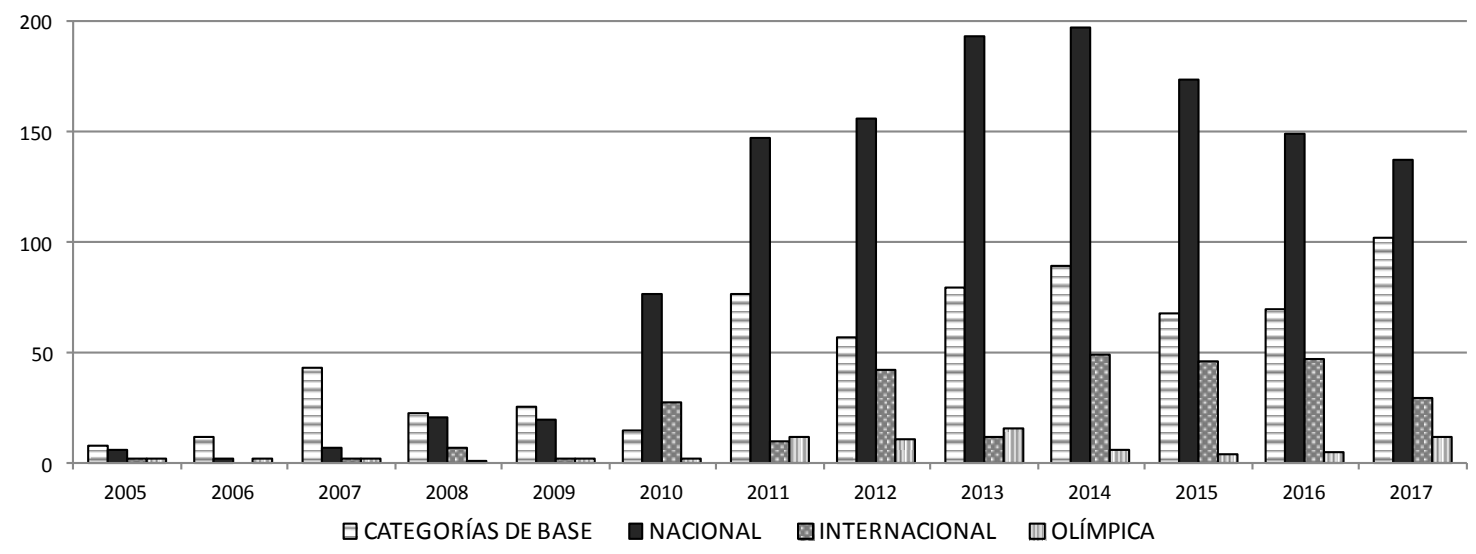

Figura 2. Número de concesiones para la natación entre 2005 y 2017 por categoría y año.

Es posible observar que hasta el año 2009 el número de atletas contemplados en las categorías de base fue mayor que el número de atletas de la categoría nacional; siendo este último, a su vez, mayor que el número de atletas de nivel internacional, y así sucesivamente. Esta configuración en la distribución de becas se ve modificada a partir de 2010, cuando se produce un aumento en el direccionamiento de becas para atletas en la categoría nacional.

En relación al total de atletas contemplados entre los años 2005 y 2017, sólo el $12 \%(n=122)$ de los mismos recibieron beca por más de tres años (Tabla 1); siendo consecutivos o no.

Tabla 1. Continuidad en la recepción del beneficio y número de atletas.

\begin{tabular}{cc}
\hline Continuidad & Número de atletas \\
\hline 1 año $(46,5 \%)$ & 473 \\
2 años $(20,5 \%)$ & 208 \\
3 años $(12 \%)$ & 122 \\
4 años $(10 \%)$ & 102 \\
5 años $(5,7 \%)$ & 58 \\
6 años $(2,9 \%)$ & 30 \\
7 años $(1,9 \%)$ & 19 \\
8 años $(0,3 \%)$ & 3 \\
9 años $(0,2 \%)$ & 2 \\
\hline Total & 1.017 \\
\hline
\end{tabular}

Se puede observar que entre 2005 y 2017 la mayoría de los atletas recibió el incentivo financiero durante apenas un año $(n=473)$. La figura siguiente presenta las posibles diferencias entre las categorías de beca de acuerdo con la continuidad de recepción del beneficio. 


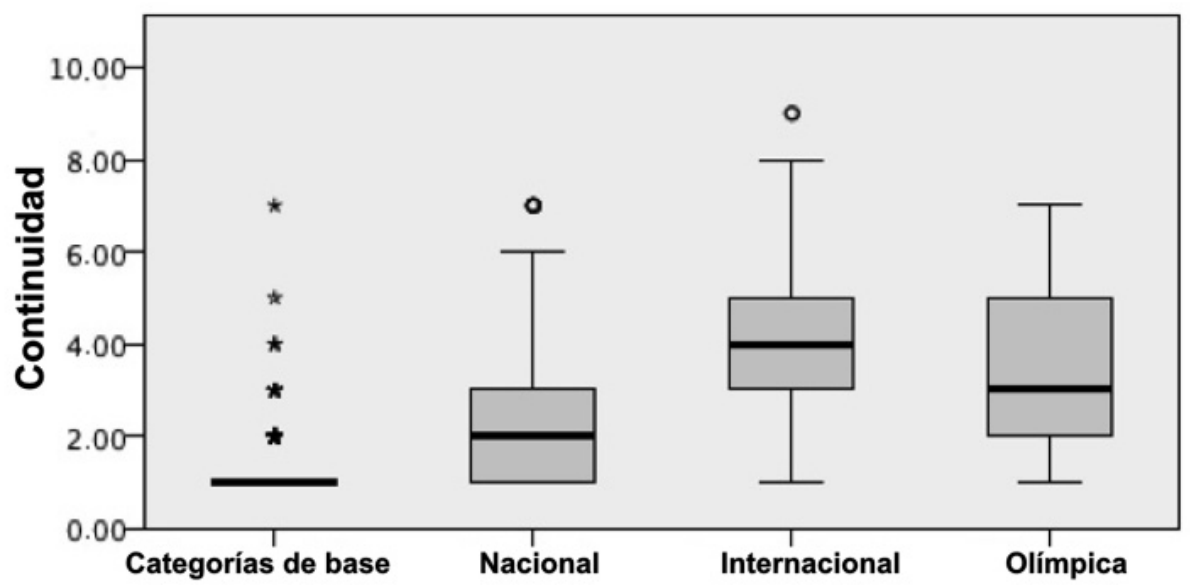

Figura 3. Diferencias entre las categorías de beca en función de la continuidad, mediante el test de Kruskal-Wallis para muestras independientes.

A partir de la prueba, se confirmó que la distribución de la continuidad presenta diferencias significativas en las respectivas categorías del programa $(p<0,001)$. Además, para identificar la magnitud del efecto de estas diferencias en general, se calculó el coeficiente épsilon al cuadrado (Kelley, 1935, Tomczak y Tomczak, 2014), obteniéndose un efecto moderado $\left(\varepsilon^{2}=0,15\right)$.

Posteriormente, se realizaron análisis post hoc con comparaciones pairwise a través del estadístico $U$ de Mann-Whitney (con la corrección de Bonferroni para los valores de la significancia). De la misma forma, se calculó la $r$ de Rosenthal (Rosenthal, 1991) en las categorías que presentaron diferencias significativas, para verificar el tamaño del efecto de las mismas (Tabla 2). Así, se puede observar el efecto más grande $(r=0,91)$ entre las becas de las categorías base y las internacionales, siendo característica la existencia de diferencias entre la continuidad en estas categorías.

Tabla 2. Análisis post hoc con comparaciones pairwise entre las diferentes categorías.

\begin{tabular}{ccccc}
\hline Categoría 1 - Categoría 2 & $\boldsymbol{U}$ & $\boldsymbol{z}$ & $\boldsymbol{p}^{\boldsymbol{*}}$ & $\boldsymbol{r}$ \\
\hline Categorías de Base - Nacional & $-221,216$ & $-11,105$ & $<0,001$ & 0,58 \\
Categorías de Base - Olímpica & $-401,359$ & $-8,625$ & $<0,001$ & 0,45 \\
Categorías de Base - Internacional & $-465,271$ & $-17,543$ & $<0,001$ & 0,91 \\
Nacional - Olímpica & $-180,143$ & $-0,549$ & $<0,001$ & 0,03 \\
Nacional - Internacional & $-244,6055$ & $-9,788$ & $<0,001$ & 0,51 \\
Olímpica - Internacional & 63,912 & 1,307 & 1,000 & 0,07 \\
\hline
\end{tabular}

*Significancia ajustada mediante corrección de Bonferroni.

Por otra parte, en la Tabla 3 aparece el número de atletas que progresaron desde la categoría de base hacia categorías superiores. Asimismo, se indica el número de atletas que retrocedieron desde la categoría olímpica hacia categorías inferiores. 
Tabla 3. Análisis de progresión y retroceso de las becas.

\begin{tabular}{cc}
\hline Progresión de los atletas & Atletas (n) \\
\hline Categorías de base & 324 \\
Categorías de base + nacional & 157 \\
Categorías de base + nacional + internacional & 54 \\
Progresión no gradual desde las categorías de base & 13 \\
\hline Retroceso de los atletas & Atletas (n) \\
\hline Olímpica & 17 \\
Olímpica + internacional & 6 \\
Olímpica + internacional + nacional & 7 \\
Sin retroceso gradual desde la categoría olímpica & 4 \\
\hline Todas las categorías & 3 \\
\hline
\end{tabular}

Como podemos observar, solamente tres nadadores recibieron el financiamiento en todas las categorías de becas, es decir, fueron financiados por el gobierno brasileño en todas las categorías (categorías de base, nacional, internacional y olímpica). Otro punto a destacar, es que el 58,8\% $(n=324)$ de los atletas con becas de base, tuvieron únicamente esta beca; sin presentar progresión para las demás categorías.

Por otro lado, cerca del 41,19\% $(n=227)$ de los atletas presentaron progresión en las becas, siendo $28,49 \%(n=157)$ para la categoría nacional, 9,80\% $(n=54)$ para la categoría internacional y $0,54 \%(n=3)$ para la categoría olímpica. Otro $2,35 \%(n=13)$ presentaron progresión, sin embargo, de modo no gradual, es decir, sin presentar una lógica constante, dejando de recibir, por ejemplo, la categoría de beca nacional y migrando directamente a la beca internacional.

En general, sólo el 5,30\% $(n=54)$ del total de atletas contemplados en estos 13 años presentó progresión en las becas de base hacia la beca internacional, y sólo el 0,29\% $(n=3)$ progresó desde la categoría de base hasta la olímpica, es decir, de cada 100 atletas contemplados, sólo cinco alcanzaron la categoría internacional; mientras que, de cada 330 atletas, apenas uno llegó a la categoría olímpica.

Del total de 37 nadadores que fueron considerados con la beca olímpica entre 2005 y 2017, cerca del 45,9\% $(n=17)$ recibieron solamente esta beca. Por otro lado, el $16,2 \% \quad(n=6)$ retrocedió desde la beca olímpica hacia la beca internacional; el 18,9\% $(n=7)$ desde olímpica a internacional y, posteriormente, nacional; el 8,10\% $(n=3)$ retrocedió hasta la categoría de base, pasando por el resto de categorías; y, por último, un 10,81\% (n=4) presentó retroceso de dos, tres o cuatro categorías; sin haberse producido de manera gradual.

\section{DISCUSIÓN}

Entre 2005 y 2017 fue posible observar un aumento significativo en la evolución de las becas, apuntando a un escenario de aumento del financiamiento en la modalidad de natación a través del Programa Bolsa-Atleta. La identificación de la constante evolución del volumen financiero de los beneficios (\$ 47.568 en 2005 y $\$ 773.664$ en 2017) evidencia la importancia de analizar la continuidad 
de los nadadores analizados en este período, buscando identificar si aquellos atletas que reciben incentivo económico en las categorías de base, evolucionan hacia categorías posteriores; produciéndose un acompañamiento efectivo en la formación de atletas de rendimiento en el país.

Los datos observados se corroboran con las afirmaciones de varios autores (Hollings, Mallett y Hume, 2014; Huxlei, O'Connor y Bennie, 2018; Rees et al., 2016), en relación al hecho de que muchos sistemas de identificación de talentos en el deporte utilizan el éxito competitivo precoz como el criterio principal en la selección de atletas, dentro de un programa de desarrollo deportivo, aun existiendo evidencias de que, en todos los niveles de desempeño deportivo, el éxito deportivo juvenil no prevé significativamente el éxito a largo plazo (Hollings, Mallett y Hume, 2014; Huxlei, O'Connor y Bennie, 2018; Rees et al., 2016).

Teniendo en consideración que el programa analizado en el presente estudio parte de los resultados obtenidos por los atletas para realizar la concesión del beneficio financiero, podemos percibir que, al no producirse la renovación o progresión en los incentivos, los resultados deportivos pueden haber sido reducidos, o simplemente dejado de existir.

En numerosas ocasiones, el apoyo financiero es de vital importancia para que los atletas no profesionales permanezcan en la práctica deportiva (Wicker et al., 2012), sin embargo, a partir de los datos obtenidos, es posible ratificar las afirmaciones de De Bosscher y De Rycke (2017), sobre la posibilidad de que un potencial número de atletas destacados pudieran ser excluidos de la recepción de incentivos financieros, si éstos se guían en los resultados deportivos, de manera exclusiva. Además, los datos apuntan a los ofrecidos por SeguíUrbaneja et al. (2020) al demostrar que las categorías básicas no siempre están directamente relacionadas con las categorías superiores.

Algunos estudios muestran que la formación y selección de talentos deportivos es un proceso lento; siendo un período aproximado de ocho a 12 años para obtener resultados relevantes (Hastie et al., 2013, Rees et al., 2016, Vieira y Vieira, 2001). Según Côté y Hancock (2016), los sujetos encargados de establecer políticas deportivas necesitan reestructurar los programas de deportes para jóvenes, con el propósito de equilibrar las políticas que maximizan los beneficios de la práctica deportiva. Asimismo, los responsables de dichas políticas, tienen la desafiante tarea de formular políticas y formas de administración de los programas de deportes para jóvenes; atendiendo las múltiples necesidades de los participantes, así como impactando de manera positiva en la tasa de participación, el desempeño deportivo futuro y el desarrollo personal de los atletas (Côté y Hancock, 2016).

Según Rees et al. (2016), la mayoría de los sistemas de identificación de talentos en el deporte utilizan el desempeño en la juventud y el éxito competitivo precoz como el principal criterio de selección en los programas de desarrollo deportivo. A partir de las evidencias, los autores recomiendan que los encargados de establecer políticas públicas para el deporte reconozcan que los primeros programas de apoyo al atleta, no son el único camino para el desarrollo de talento, por lo tanto, esto debería ser una prioridad para futuras investigaciones. 
En cuanto al programa brasileño analizado, algunos estudios apuntan que sería equivocado afirmar que el beneficio sea responsable de la mejora en el desempeño deportivo de los atletas exclusivamente (Guimarães, 2009, Dias et al., 2016; Moraes e Silva, Mezzadri, de Souza y de Souza, 2015; Ordonhes et al., 2016); teniendo en cuenta que el programa debería establecerse a partir de una planificación a largo plazo, y no como una política de causa y efecto.

En relación al total de los nadadores que progresaron desde las becas de base hacia la beca internacional, 14 atletas participaron de campeonatos mundiales entre 2015 y 2017; principal competición internacional de la modalidad. En cuanto a los nadadores que progresaron de las categorías de base hacia la categoría olímpica, los tres atletas participaron en mundiales, eventos panamericanos, sudamericanos y Juegos Olímpicos (JJ. OO.).

En la lista de atletas contemplados entre los años 2005 y 2017, es posible percibir que algunos de los mismos forman parte de la actual selección brasileña de natación. Así de entre los 17 que recibieron solamente beca olímpica, encontramos 10 atletas pertenecientes a la selección (58\%). En cuanto a los atletas que presentaron progresión desde la categoría de base hasta la categoría internacional, observamos que de los 54, 10 forman parte de la selección brasileña $(18,51 \%)$.

Los resultados significativos que se observaron en los nadadores de categorías superiores que no recibieron la promoción en categorías inferiores, puede invitar a la reflexión sobre la forma en la que se distribuyen los fondos públicos en Brasil, teniendo como requisito central los resultados inmediatos a corto plazo; puesto que basándose en los mismos, exclusivamente, podría no ser suficiente para garantizar la progresión futura del atleta, existiendo otros factores determinantes para la formación de un atleta (Blázquez y Feu, 2012; Barker-Ruchti et al., 2018; Côté y Hancock, 2016; Rees et al., 2016).

A partir del análisis de los datos, es posible realizar aproximaciones con el trabajo de Barker-Ruchti et al. (2018), formulando cuestiones en relación a la entrega de recursos públicos para la práctica deportiva; instando a la reflexión en cuanto a si resulta ideal la forma en que se produce el reparto de dichos recursos para la formación de atletas en el país.

De este modo, se propone que el direccionamiento de recursos financieros se realice de forma directa a los aspectos específicos relacionados con la formación deportiva, o sea, destinando a materiales y equipamientos deportivos, gastos de viajes, entrenamientos y competiciones de alto nivel, entre otros. Así, se pueden considerar las especificidades de cada modalidad.

A partir de los resultados observados, podemos percibir que no existe todavía un modelo de gestión deportiva que considere el deporte como elemento político de gestión que necesite ser pensado a corto, mediano y largo plazo; siendo necesario tener en consideración la planificación a largo plazo (Saretta et al., 2015). Las acciones dirigidas, los beneficios e incentivos financieros, en numerosas ocasiones presentan un carácter inmediato; por ello, se precisa de 
una propuesta gubernamental de incentivo al deporte dirigida a un proceso de calificación de los atletas y de desarrollo de las modalidades, que no acabe quedando en segundo plano.

El hecho de que este estudio analice los atletas contemplados por una política de financiamiento deportivo (Programa Bolsa-Atleta) en una sola modalidad (natación) caracteriza la limitación de la investigación. En este sentido, recomendamos la realización de otras investigaciones relacionadas con las políticas deportivas dirigidas a los atletas, contribuyendo a la formulación y aplicación de políticas públicas en diversas esferas internacionales. Además, resultaría interesante el análisis de otras modalidades deportivas individuales, para poder comparar con los resultados observados en la muestra ahora elegida.

\section{CONCLUSIONES}

El presente estudio tuvo como objetivo identificar si los nadadores brasileños que reciben incentivos financieros en las categorías de base continúan su deporte hasta las categorías de financiamiento más altas en el programa: internacional y olímpico.

Se puede percibir que el financiamiento público realizado en las categorías de base no representó garantía de permanencia de los nadadores en el deporte. También fue posible identificar atletas que presentaron buenos resultados en categorías superiores del programa sin necesariamente aparecer en la lista de contempladas iniciales. Los datos observados nos llevan a cuestionar dónde estaban en años anteriores esos atletas y si éstos fueron contemplados con otros incentivos (públicos, privados u otras formas de mantenimiento, como, por ejemplo, la financiación familiar).

A partir de los datos obtenidos en el presente estudio se puede cuestionar si la forma en cómo se produce el traspaso de fondos es la ideal para la formación de atletas en el país en cuestión. Aunque fue posible observar inversiones más prominentes en natación a partir de 2010, se propone que haya una descentralización de los recursos pensados a largo plazo, considerando un proceso de calificación de los nadadores y las especificidades de la modalidad en cuestión, con el objetivo de dirigir los recursos de forma más efectiva.

\section{REFERENCIAS BIBLIOGRÁFICAS}

Barker-Ruchti, N., Schubring, A., Aarresola, O., Kerr, R., Grahn, K., \& McMahon, J. (2018). Producing success: A critical analysis of athlete development governance in six countries. International Journal of Sport Policy and Politics, 10(2), 215-34. https://doi.org/10.1080/19406940.2017.1348381.

Blázquez, A., \& Feu, S. (2012). Motivación en actividad física mantenimiento en mujeres de municipios pequeños / Motivation for the practice of keep-fit physical activities for small town women. Revista Internacional de Medicina y Ciencias de la Actividad Física y el Deporte, 12(47), 571-88. Recuperado de: http://cdeporte.rediris.es/revista/revista47/artmotivacion304.htm 
Brasil (2020). Lei $n^{\circ}$ 10.891, de 9 de julho de 2004. Recuperado de: http://www.planalto.gov.br/ccivil 03/ ato2004-2006/2004/lei/l10.891.htm

Diário Oficial da União (2020). Imprensa Nacional do Brasil - Diário Oficial da União. Recuperado de: http://www.in.gov.br/consulta

Caetano, C. I., Sentone, R. G., López-Gil, J. F., Caetano, H. B. S., \& Cavichiolli, F. R. (2020). Influência do tamanho e densidade populacional no rendimento esportivo da ginástica artística brasileira (Influence of population size and density on sports performance of Brazilian artistic gymnastics). Retos, 38, 6670. Recuperado de: https://recyt.fecyt.es/index.php/retos/article/view/74338

Cohen, J. (1988). Statistical Power Analysis for the Behavioral Sciences (2a ed.). Hillsdale (Nueva Jersey), EE. UU.: Lawrence Erlbaum Associates.

Côté, J., \& Hancock, D. J. (2016). Evidence-based policies for youth sport programmes. International Journal of Sport Policy and Politics, 8(1), 51-65. https://doi.org/10.1080/19406940.2014.919338

De Bosscher, V., Bingham, J., Shibli, S., van Bottengurg, M., \& De Knop, P. (2008). The global sporting arms race: An international comparative study on sports policy factors leading to international sporting success. Oxford, Reino Unido: Meyer \& Meyer Sport.

De Bosscher, V. \& De Rycke, J. (2017). Talent development programmes: a retrospective analysis of the age and support services for talented athletes in 15 nations, European Sport Management Quarterly, 17(5), 590-609. https://doi.org/10.1080/16184742.2017.1324503

De Bosscher, V., Shibli, S., \& Weber, A. C. (2019). Is prioritisation of funding in elite sport effective? An analysis of the investment strategies in 16 countries. European Sport Management Quarterly, 19(2), 221-43. https://doi.org/10.1080/16184742.2018.1505926

De Bosscher, V, De Knop, P, Van Bottenburg, M, Shibli, S, \& Bingham, J. (2009). Explaining international sporting success: An international comparison of elite sport systems and policies in six countries. Sport Management Review, 12(3), 113-36. https://doi.org/10.1016/j.smr.2009.01.001

De Bosscher, V., De Knop, P., Van Bottenburg, M., \& Shibli, S. (2006). A conceptual framework for analysing sports policy factors leading to international sporting success. European sport management quarterly, 6(2), 185-215. https://doi.org/10.1080/16184740600955087

Dias, Y. R., Moraes e Silva, M., Figuerôa, K. M, Nunes, R. J., Rojo, J. R, \& Mezzadri, F. M. (2016). O Judô no programa governamental Bolsa-Atleta: a distribuição espacial dos bolsistas (2011-2013). Pensar a Prática, 19(1), 11829. https://doi.org/10.5216/rpp.v19i1.37897

Digel, H. (2002). A comparison of competitive sport systems. New Studies in Athletics, 17(1), 37-50. Recuperado de: https://www.iaaf.org/download/downloadnsa?filename=09f854af-b4ac-42eab127-52e1fdf71622.pdf\&urlslug=a-comparison-of-competitive-sport-systems

Feiler, S., Wicker, P., \& Breuer, C. (2018). Public subsidies for sports clubs in Germany: funding regulations vs. empirical evidence. European Sport Management $\quad$ Quarterly, 1-21. https://doi.org/10.1080/16184742.2018.1541915

Fernández, J. E. R., Couto, J. M. P., \& Aza, E. T. (2016). La gestión del deporte en clave educativa de Motricidad Humana (The management of the sport from 
an educational perspective in Human Kinetics). Retos, 30, 92-97. Recuperado de: https://www.redalyc.org/pdf/3457/345744747017.pdf

Gimeno, F., Garzo, C., Velasco, I., Sáenz, A. \& Gutiérrez, H. (2017). Evaluación de características psicológicas implicadas en el desempeño de jóvenes gestores deportivos / Psychological Characteristics Assessment Involved in Youth Sports Managers' Performance. Revista Internacional de Medicina y Ciencias de la Actividad Física $y$ el Deporte, 17(66), 335-47. https://doi.org/10.15366/rimcafd2017.66.008

Guimarães, A. S. (2009). A bolsa-atleta eleva o desempenho de seus beneficiários? Análise do período 2005-2008. Recuperado de: https://www.researchgate.net/profile/alexandre guimaraes12/publication/260 400725 a bolsaatleta eleva o desempenho de seus beneficiarios analise do periodo 2 005-2008/links/584a92ec08aee436cbff067e/a-bolsa-atleta-eleva-odesempenho-de-seus-beneficiarios-analise-do-periodo-2005-2008.pdf

Green, M., \& Oakley, B. (2001). Elite sport development systems and playing to win: uniformity and diversity in international approaches. Leisure Studies, 20(4), 247-67. https://doi.org/10.1080/02614360110103598

Hastie, P. A., Calderón, A., Rolim, R. J., \& Guarino, A. J. (2013). The development of skill and knowledge during a sport education season of track and field athletics. Research quarterly for exercise and sport, 84(3), 336-44. https://doi.org/10.1080/02701367.2013.812001

Hernández-Álvarez, J. L., Velázquez-Buendía, R., Martínez-Gorroño, M.E, \& Díaz del Cueto, M. (2010). Creencias y perspectivas docentes sobre objetivos curriculares y factores determinantes de actividad física. Revista Internacional de Medicina y Ciencias de la Actividad Física y el Deporte, 10(38), 336-55. Recuperado

de: Http://cdeporte.rediris.es/revista/revista38/artcrencias160b.htm

Hollings, S. C., Mallett, C. J., \& Hume, P. A. (2014). The transition from elite junior track-and-field athlete to successful senior athlete: why some do, why others don't. International Journal of Sports Science \& Coaching, 9(3), 457-71. https://doi.org/10.1260/1747-9541.9.3.457

Houlihan, B, \& Green, M. (2008). Comparative Elite sport development, systems, structures and public policy (1 $\left.{ }^{\mathrm{a}} \mathrm{ed}\right)$. Amsterdam, Holanda: Elsevier Ltd.

Huxley, D. J., O'Connor, D., \& Bennie, A. (2018). Olympic and World Championship track and field athletes' experiences during the specialising and investment stages of development: a qualitative study with Australian male and female representatives. Qualitative Research in Sport, Exercise and Health, 10(2), 256-272. https://doi.org/10.1080/2159676X.2017.1393452

Inteligência Esportiva (2018). Banco de Dados do projeto Inteligência Esportiva. Recuperado de: http://www.inteligenciaesportiva.ufpr.br/site/.

Kelley, T. L. (1935). An Unbiased Correlation Ratio Measure. Proceedings of the National Academy of Sciences of the United States of America, 21(9), 554-9. Recuperado de: https://www.ncbi.nlm.nih.gov/pmc/articles/PMC1076656/pdf/pnas017610040.pdf

Kothari, C. R. (2004). Research methodology: Methods and techniques. Nueva Delhi, India: New Age International. 
Leiva, A., Sánchez, A. \& Martínez, M. J. (en prensa). Impact Analysis of Ado Plan in the Spanish Olympic Results. Revista Internacional de Medicina y Ciencias de la Actividad Física $y$ el Deporte. Recuperado de: http://cdeporte.rediris.es/revista/inpress/artanalisis1277.pdf

Martínez de Ojeda, D., Puente-Maxera, F. y Méndez-Giménez, A. (en prensa). Efectos motivacionales y sociales de un programa plurianual de educación deportiva / Motivational and Social Effects of a Multiannual Sport Education Program. Revista Internacional de Medicina y Ciencias de la Actividad Física y el Deporte. Recuperado de: http://cdeporte.rediris.es/revista/inpress/artefectos1193e.pdf

Moraes e Silva, M., Mezzadri, F. M, de Souza, D. L, \& de Souza, P. M. (2015). O financiamento público do rugby brasileiro: a relação governo federal e Confederação Brasileira de Rugby. Journal of Physical Education, 26(2), 21322. https://doi.org/10.4025/reveducfis.v26i2.24586

Murray, D. (2009). Reflections on Public Funding for Professional Sports Facilities. Journal of the Philosophy of Sport, 36(1), 22-39. https://doi.org/10.1080/00948705.2009.9714743

Ordonhes, M. T., da Luz, W. R., \& Cavichiolli, F. R. (2016). Possíveis relações entre investimentos públicos e obtenção de resultados: o caso da natação brasileira. Motrivivência. 28(47), 82-95. https://doi.org/10.5007/21758042.2016v28n47p82

Rosenthal, R. (1991). Meta-analytic procedures for social research (2a ed.). California, EE. UU.: Sage Publications.

Rees, T, Hardy, L, Güllich, A, Abernethy, B, Côté, J, Woodman, T, \& Warr, C. (2016). The great British medalists project: a review of current knowledge on the development of the world's best sporting talent. Sports Medicine, 46(8), 1041-58. https://doi.org/10.1007/s40279-016-0476-2

Saretta, C. B, de Carvalho, F. G, de Carvalho, B. E., \& Martins, W. D. (2015). Avaliação do desenho institucional, dos recursos e das capacidades para concretização do planejamento de longo prazo no Brasil (No. 2129). Texto para Discussão, Instituto de Pesquisa Econômica Aplicada (IPEA). Recuperado de: https://repositorio.ipea.gov.br/handle/11058/5385

Sentone, R.G, López-Gil, J. F., Caetano, C. I., \& Cavichiolli, F. R. (2019). Relationship between human development index and the sport results of Brazilian swimming athletes. Journal of Human Sport and Exercise, 14(Proc5), S2009-18. https://doi.org/10.14198/jhse.2019.14.Proc5.22

Seguí-Urbaneja, J., Inglés, E., Alcaraz, S. \& De Bosscher, V. (2020) Sport Pyramid Metaphor: Trickle Down and Up Effect in Spain. Revista Internacional de Medicina y Ciencias de la Actividad Física y el Deporte, 77(20), 1-20. http://doi.org/10.15366/rimcafd2020.77.001

Tomczak, M., \& Tomczak, E. (2014). The need to report effect size estimates revisited. An overview of some recommended measures of effect size. Trends in Sport Sciences, 21(1), 19-25. Recuperado de: http://www.wbc.poznan.pl/Content/325867/5 Trends Vol21 $2014 \% 20$ no1 20.pdf.

Vieira, L. F., \& Vieira, J. L. (2008). Talentos esportivos: estudo dos atributos pessoais dos atletas paranaenses do atletismo. Journal of Physical Education. 12(1), 7-17. Recuperado http://eduem.uem.br/ojs/index.php/RevEducFis/article/viewArticle/3759 
Wicker, P., Breuer, C., \& von Hanau, T. (2012). Is it profitable to represent the country? Evidence on the sport-related income of funded top-level athletes in Germany. Managing Leisure, 17(2-3), 221-38. https://doi.org/10.1080/13606719.2012.674396

Número de citas totales I Total references: 39 (100\%)

Número de citas propias de la revista / Journal's own references: 5 $(12,82 \%)$

Rev.int.med.cienc.act.fís.deporte - vol. 21 - número 83 - ISSN: 1577-0354 